\title{
On Proto-Languages and Archaeological Cultures: pre-history and material culture in the Tukanoan Family
}

Thiago Chacon ${ }^{1}$

\section{Resumo}

Este trabalho analisa a história cultural das línguas da família Tukano ao propor a reconstrução de 107 palavras relacionadas à cultura material comum a povos indígenas amazônicos, em especial do noroeste amazônico. A análise do sistema terminológico das palavras que podem ser reconstruídas para o Proto-Tukano, bem com as palavras que somente são reconstruídas para protolínguas intermediárias ou palavras que não podem ser reconstruídas para nenhuma protolíngua permite traçar inferências culturais sobre a evolução histórica da família Tukáno, o que é feito em diálogo com a literatura etnográfica e arqueológica do noroeste amazônico, bem como seguindo em linhas gerais as propostas de correlação entre Linguística Histórica e Arqueologia em diferentes partes do globo. Conclui-se que houve um processo claro de diferenciação cultural entre os dois ramos principais da família Tukáno, como reflexo da integração distinta dos dois ramos da família em diferentes subsistemas interétnicos regionais no noroeste amazônico.

Palavras-Chave: Família Tukano. Noroeste Amazônico. Cultural Material. Linguística Histórica, Arqueologia.

\begin{abstract}
This work analyzes the cultural history of the Tukanoan family by attempting the reconstruction of 107 words related to the material culture shared by Amazonian peoples, especially in the Northwest Amazon. The analysis of the terminological system of words that can be reconstructed to Proto-Tukanoan, as well as words that can only be reconstructed to intermediate proto-languages or words that cannot be reconstructed at all allows for a set of cultural inferences regarding the historic evolution of Tukanoan family, which is accomplished along a dialogue with the ethnographic and archeological literature of the Northwest Amazon, as well as following in general terms the proposals for linking Historical Linguistics and Archaeology in different parts of the globe. It is concluded that there was a process of cultural differentiation between the two main branches of the Tukanoan family, as the reflex of distinct integration of each branch in different regional subsystems in the Northwest Amazon.
\end{abstract}

Keywords: Tukanoan Family. Northwest Amazon. Material Culture. Historical Linguistics, Archaeology.

1 Universidade Católica de Brasília. 


\section{Introduction}

This is a preliminary investigation about the pre-history of the Tukanoan family, whose languages are currently spoken in the Northwest Amazon (cf. Map 1 below). It is an attempt to describe in linguistic terms the cultural differentiation processes that occurred from Proto-Tukanoan, and especially between the two main branches of the family, Western Tukanoan (WT) and Eastern Tukanoan (ET). 107 vocabulary items related to the material culture items of indigenous groups in the Amazon, specially the Northwest Amazon, were attempt to be reconstructed. The results of reconstructions, non-reconstructions and the analysis of the terminological system of the vocabulary of Proto-Tukanoan and intermediate proto-languages are compared to ethnographic descriptions and to the picture that archaeology and related pre-historical sciences have about the pre-historic Amazon in the last 2 millennia. As a preliminary experiment, this paper aims at contributing to Amazonian ethnology by providing more specific and testable results that can be correlated with current and future findings in related ethnological sciences.

As it will be shown, the society that spoke Proto-Tukanoan can be characterized by the following major cultural traits:

i. A more in-land than riverine life style.

ii. Game animal as a more important source of food than fish.

iii. Knowledge of different domesticated crops, including manioc, but without a specialized economy around the bitter manioc.

iv. A diverse knowledge of forest products, especially palms and varied sources of food.

v. Evidence of technology for weaving, planting, ceramics and poisoning for obtaining fish and game.

vi. A limited use of utensils, mostly ceramics, hammocks, baskets, blowgun, but no clear evidence for axe, paddle, canoes or fish traps.

In comparison with present-day Tukanoan societies the picture that emerges is of a cultural differentiation process from Proto-Tukanoan. Some explanations of such a cultural shift in the pre-history of Tukanoans are addressed, such as the formation of regional systems and the happenings documented in different archaeological sites in the Amazon around the beginning of the second millennium $\mathrm{AD}$.

The figure 1 below gives the classification of the Tukanoan family as proposed by Chacon (forthcoming), divided in two major branches: Eastern Tukanoan (ET) and Western Tukanoan (WT). Map 1 shows the geographic distribution of Tukanoan languages (the left-most set of "snowflakes" represent WT languages and the rightmost set, ET languages). 
FIGURE 1

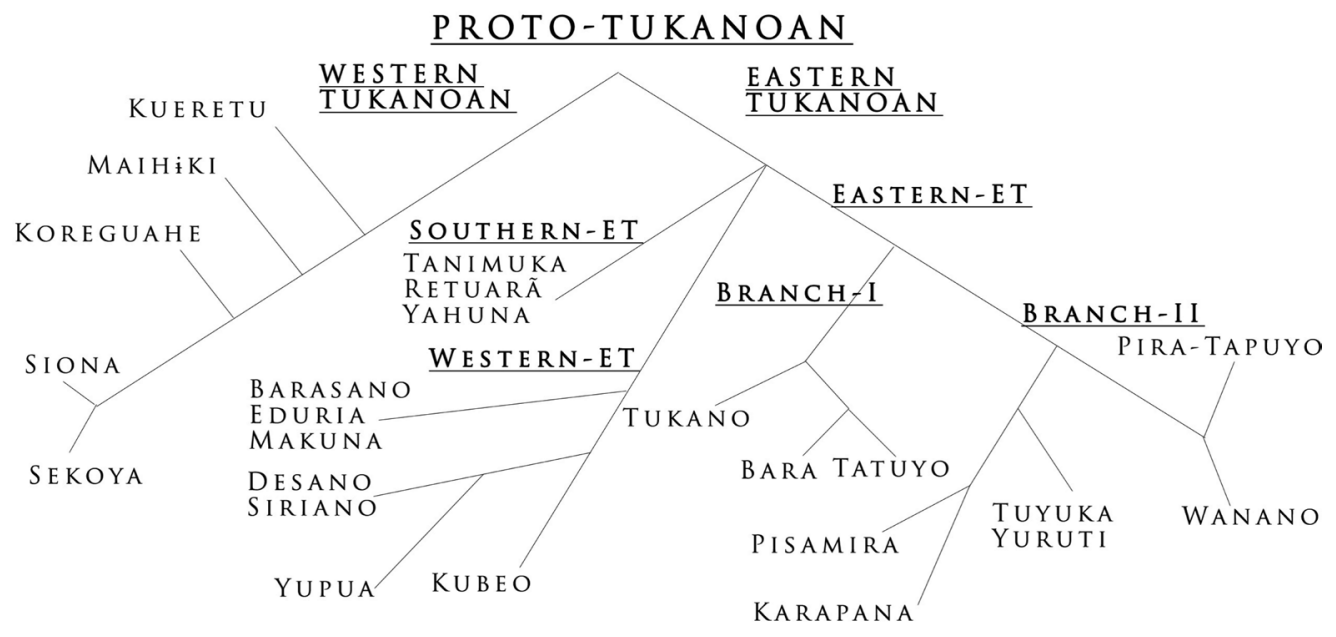

MAP 1

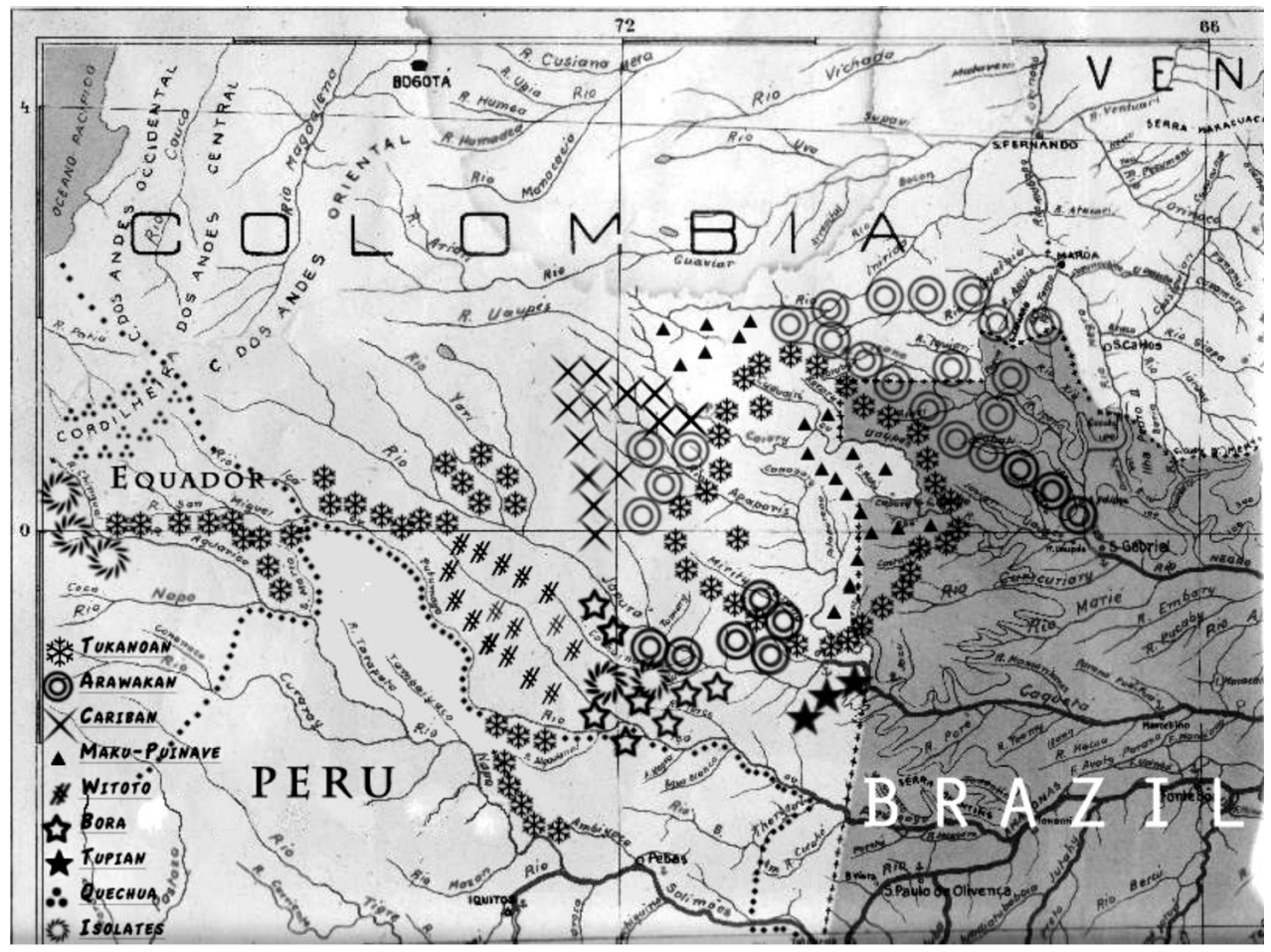


The organization of this paper is the following: in section 2, I will discuss the framework I am using for linking archaeology and linguistics, and also the current state of our knowledge about the archaeology of the Amazon and its relation to linguistics. In section 3 I present the analysis regarding the attempt at the reconstruction of the 107 material cultural items. Section 4 is the conclusion.

\section{Linking Languages and Archaeological Cultures}

Linking archaeology and languages is a process that in general involves the demonstration of a correlation of cultural traits found in the archaeological record, the reconstruction of the vocabulary referring to the material culture of a proto-language and the geographic match between the distribution of an archaeological set of cultural traits in time and space and the putative homeland of a proto-language or geographical dispersal of languages. Because one cannot have access to culture itself from the analysis of proto-languages, it is necessary that we focus on cultural traits, which are more easily comparable. Cultural history is then the result of how one correlate Historical Linguistics, Archaeology and Anthropology, where each discipline needs to be kept relatively independent and researchers ought to work in collaboration in creating a sound narrative (cf. Hornborg and Hill 2011).

For that purpose, I will comment on three case studies of established correlations between languages and archaeological set of cultural traits in different parts of the world. These studies are inspirational to this paper in the sense that I will follow their conceptions for linking languages and archaeological cultural traits to the fullest, except that my analysis of the vocabulary items will focus more on the analysis of whole terminological systems, rather than whether an item can be reconstructed (as evidence that the proto-language society had that particular item) or cannot be reconstructed.

The case of the Lapita culture and the Oceanic linguistic subfamily (from the Austronesian family) in eastern Oceania and southern Pacific is based on the observation of the patterns of expansion of an archaeological set of cultural traits into new territory and the corresponding evidence of the expansion of languages into the same territory. The Lapita expansion in parts of Oceania is very clear both in areas of previous human occupation or in pristine islands (Kirch 2000). Given the relative uniformity of Lapita cultural traits when compared to earlier records, it is clear that there was an expansion of a different type of culture that had never been seen in those areas before (Ross and Osmond 2007). Linguistically, such an expansion can be matched with the arrival of the ancestor language of the Oceanic family. Some elements of the Lapita culture match the material culture vocabulary that can be reconstructed to Proto-Oceanic, while many aboriginal languages of those 
areas seem to have borrowed words referring to those particular vocabulary items (e.g. the term of 'pig', Blust 1976).

The case of the Olmec culture and the Mixe-Zoquean family in Mesoamerica provides an interesting case where key elements in the Mesoamerican cultural area have been borrowed from Mixe-Zoquean languages into unrelated languages in the zone (Campbell and Kaufman 1976). Given that the homeland of Proto-Mixe-Zoquean and the earliest Olmec sites correlate (Campbell and Kaufman 1976), one is tempted to suggest that the Olmec were a single people that spoke Proto-Mixe-Zoquean and diffused their cultural patterns for all other cultures in Mesoamerica. However, archaeologists have long debated whether the Olmecs in the Formative Period (from $2500 \mathrm{BC}$ to $200 \mathrm{AD}$ ) should be thought as some kind of Mother Culture, or that "Olmecs" may actually refer to a cultural complex of the Formative Period in Mesoamerica and is itself the result of multilateral contributions (cf. Blomster 2005, Pye 2006, Sharer et al. 2006). Such a debate is also well founded in linguistic terms. The concept of Mesoamerica as a linguistic and cultural area is itself an indication of multilateral diffusion. Also, many words referring to key elements in Mesoamerican cultural area cannot be fully reconstructed to Proto-Mixe-Zoquean, but actually to lower branches in the family. So, it is possible that within the Olmec cultural complex, speakers of Mixe-Zoquean languages have been important sources of cultural items, but not the only source, strengthening the argument for the multilateral diffusion hypothesis.

Finally, the Proto-Indo-European case is a seminal one in that there is a whole set of complex cultural traits reconstructed to the proto-language, but they cannot be matched safely with an archaeological culture, given that linguists have disagreed on the exact location of a Proto-Indo-European homeland and the numerous similar "cultures" that can be found in the archaeological record matching the material culture vocabulary for ProtoIndo-European (cf. Mallory's 1991). On the other hand, as soon as one can define the Proto-Indo-European homeland based on independent linguistic evidence, it is clear that the expansion of the Indo-Europeans can be matched with the expansion of an archaeological culture from the Steppes of the Caspian sea known as Kurgan. The connection between the Kurgan and Proto-Indo-Europeans is also based on patterns of dispersion of Kurgan sites in Europe that correlated with the expansion of Indo-European languages and the existence of material culture in the Kurgan records that matches the reconstructed vocabulary for Proto-Indo-European.

\subsection{The Pattern of Languages and Archaeological Cultures in the Amazon}

Archaeologists have often tried to correlate the pattern of diffusion of three widest distributed language families in the Amazon - Cariban, Arawakan 
and Tupian - with the also wide distribution of ceramic traditions. While the Cariban family has been suggested to correspond to the Incised Punctuated Tradition, given the almost exclusive eastern Amazonian distribution of both the family and the pottery tradition (cf. Neves 2008), the association of Arawakan and Tupian families with ceramic traditions are more complex.

The Polycrhome Tradition has been associated with the Tupian family given that the upper Madeira river is where the oldest type of Polychrome pottery is found and also where is located the putative homeland of ProtoTupi (cf. Rodrigues 1964). Other types of association are somewhat more indirect, based on analogical patterns between the Polychrome expansion in the Amazon river valley and in the Atlantic shore and expansion of Tupian groups in the same areas (Neves 2008). ${ }^{2}$

Association of Arawakan family and the Incised Rim tradition is much more problematic. It is based on a chronology of two related types of pottery: the Barrancoid tradition, with origin in Northern Colombia, followed by the Incised Rim in the central Amazon area in areas of traditional Arawakan occupation (cf. Neves 2011). There seems to exist a consensus that the homeland of the Arawakan family is somewhere between the Orinoco and Rio Negro basins (Hill and Santos-Granero 2002). That being the case, it would be likely that Arawakan speakers could be directly involved in the integration of the Central Amazon and Northern Colombia areas as suggested by the distribution of the Barrancoid/Incised Rim pottery traditions (see also Hornborg 2005).

The general archaeological chronology of the Amazon shows that around the first year of the present era (after a long gap in the mid-Holocene) until the beginning of the second millennium there was a moment of great population growth and cultural differentiation in separate geographic areas (cf. Neves 2011). Agriculture was intensified in this time, as it is evident by the appearance of anthropogenic dark soils, although still a diversified type of subsistence was based on the exploration of diverse ecological resources. Following this period, in the beginning of the second millennium of the current era, one observes an expansion of the polychrome tradition, which replaced in many places previous, more regional ceramic traditions. In many places in the central Amazon, structural defenses were built around villages, indicating that the expansion of the polychrome tradition was likely accompanied by an intensification of warfare (cf. Neves 2011).

This chronology is also found in the Araracuara area, in the middle Caquetá river (Northwest Amazon), an area of particular interest for the understanding

2 It is clear that one lacks enough linguistic evidence to make these correlations more concrete. This is probably the reason why linguists have often been silent with respect to associations between pottery traditions and languages. 
of Tukanoan pre-history. In this site, the appearance of the polychrome tradition around the second millennium has been found in connection with anthropogenic black soil and other indications of agricultural intensification and material cultural complexification, showing an abrupt change in the archaeological record from an early, more localized and more simplified type of pottery (Oliver 2008). Such early record indicates a previous society that has domesticated plants, but still did not rely intensively on agriculture, making more use of diversified economic resources in their environment (Herrera et. al. 1992).

Although the spreading of languages and archaeological cultural traits are usually attempted to be correlated based on demic migrations and economic propelling forces, such as agriculture (cf. Lathrap 1970, Bellwood \& Renfrew 2003), more recently Amazonian ethnologist have focused in other dynamics of dispersal of languages and cultural traits. As Neves (2011) analyzes, given that many groups had a similar knowledge of agriculture at the same historical period in the first millennia of the present era, it is likely that expansion of major language families in the Amazon need further elements to be fully explained. Furthermore, there is a hypothesis that before colonial times a unique agricultural system has never developed in the Amazon (Denevan 2001), while pre-colonial Amazonian societies had agriculture as part of their diversified economy, they were distinct from "agriculturalist" societies.

Neves (2011) suggest that the polychrome tradition distribution might be the result of Tupi-Guarani speaking groups migrations motivated by warfare, captive-taking and cannibalism. On the other hand, Hornborg (2005) and Hornborg and Hill (2011) suggest that the expansion of Arawakan languages and cultural traits associated to an Arawakan ethos (cf. Hill and Santos-Granero 2002 ) is the result of the formation of local and supra-local interethnic systems, motivated by the Arawakan emphasis on commerce, ritual, spatial and social networks, likely accompanied by ethnogenetic processes of redefinition of local identities, cultures and languages.

My analysis, to be developed in the next two sections, indicates that ProtoTukanoan speakers had a cultural pattern similar to the archaeological cultures that appeared around the beginning of the present era, and that a major cultural differentiation processes occurred in more recent times and distinctly for different languages of the family after an initial split between two major branches, ET and WT. This scenario first correlates with the facts described in the Araracuara area in the middle Caquetá river, an area adjacent to where I propose the Proto-Tukanoan homeland (see also Chacon forthcoming). Second, it correlates with the geographical dispersal and integration of WT and ET in the formation of distinct regional systems in the Northwest Amazon. 


\section{The Vocabulary of Proto-Tukanoan Basic Material Culture}

Results and Methods: words for 107 material items of traditional Northwestern Amazonian cultures were analyzed in Tukanoan languages. ${ }^{3}$ Items correspond to one of 10 semantic domains: WEAVING, AGRICULTURE, JUNGLE RESOURCES, RIVER RESOURCES, UTENSILS, PALMS AND TREES, INSECTS AND OTHER FOODS, RITUALISTIC MATERIALS, MANIOC COMPLEX and SOCIAL SPACE. Items were matched semantically with one or more words in the Tukanoan languages, followed by the creation of cognate sets and reconstruction of lexical items into Proto-Tukanoan (PT), Proto-Eastern-Tukanoan (PET) and Proto-Western-Tukanoan (PWT) was attempted, following Chacon (forthcoming), which provides a reconstruction of the consonantal system of PT and classification of the Tukanoan family distinct to Waltz and Wheeler (1972), Malone (1987) and Barnes (1999).

Table 1 below summarizes the results. ${ }^{4}$ The results of the reconstructions were accompanied by observations in the right-most column regarding etymology, semantic relations to other items and distribution of cognates. When an item cannot be reconstructed to a particular branch or to PT, it was marked with a darker cell. Items were also classified according to the consistency of proto-languages reflexes. The sign "\%", followed by the particular family branch abbreviation, \%ET, \%WT and \%ET\&WT for both branches, indicating an inconsistent set of reflexes, was placed under the word for the item in English. The particular reasons why the reflexes are inconsistent were highlighted in the observation column, and are developed in the discussion session of this study.

3 The data is from about 22 Tukanoan languages, from the aprox. 30 languages reported for the family. Many are extinct already (cf. Chacon forthcoming). The data was taken from dictionaries (which ranges from about 800 to 3500 words) available for most Tukanoan languages that members of SIL have worked on (see the bibliography for a complete list), a robust dictionary of Tukano (Ramirez 1997a), an excellent Colombian publication with basic phonological and grammatical information on most Tukanoan languages and the Swadesh list of 200 words for each language (González de Pérez and Rodríguez de Montes, 2000), a very good SIL publication with the complete Swadesh and Rowe wordlist plus additional words for most indigenous languages in Colombia (cf. Huber and Reed, 1992), and Koch-Grünberg wordlists (1913 and 1914), which are extremely valuable resources on extinct Tukanoan languages.

4 Language name abbreviations used in this paper are: TAN Tanimuka, BAS Barasano, Kub Kubeo, DEs Desano, tuk Tukano, wan Wanano, Kue Kueretu, MAI Maihiki, kor Koreguahe, sEk Sekoya and sio Siona. 


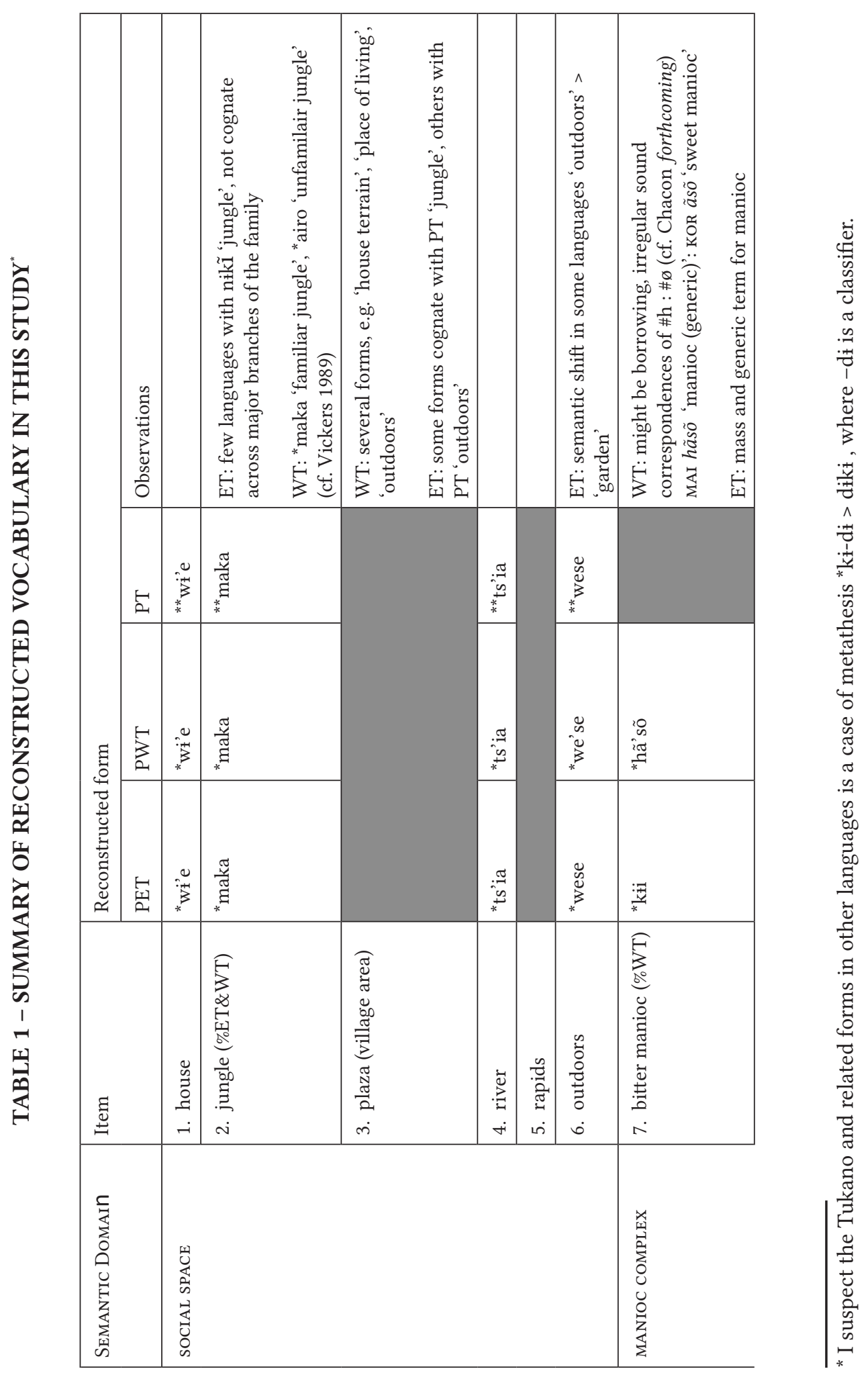




\begin{tabular}{|c|c|c|c|c|c|c|c|c|c|c|c|c|}
\hline & 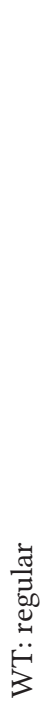 & 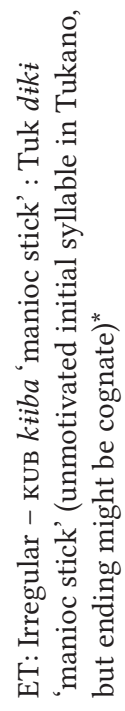 & 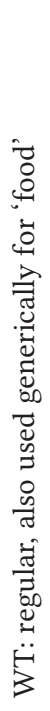 & 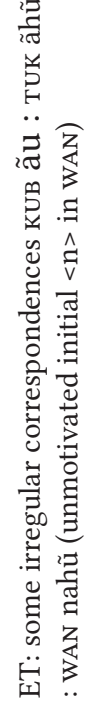 & 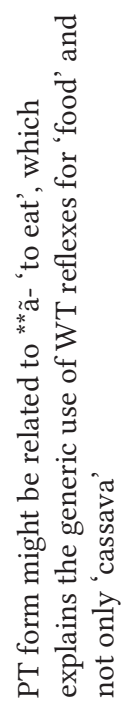 & 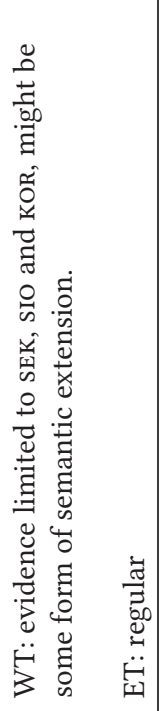 & & & & & & 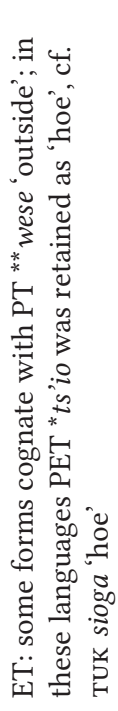 \\
\hline & $\frac{\text { 涍 }}{*}$ & & 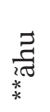 & & & & & & & & & 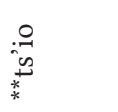 \\
\hline & $\frac{i \sqrt{*}}{*}$ & & $\underset{\substack{\pi \\
*}}{\vec{E}}$ & & & 足 & & & & & & $\underset{*}{\stackrel{0}{\infty}}$ \\
\hline & $\frac{\text { 㴗 }}{*}$ & & 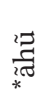 & & & $\begin{array}{l}\frac{2 \pi}{20} \\
3 \\
* \\
*\end{array}$ & & & $\begin{array}{l}\text { io } \\
\text { io } \\
\text { * }\end{array}$ & & $\begin{array}{l}\text { चँ } \\
\frac{\pi}{\pi} \\
\frac{\pi}{*}\end{array}$ & 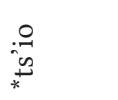 \\
\hline 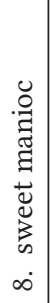 & 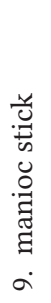 & & 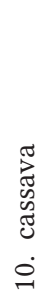 & & & 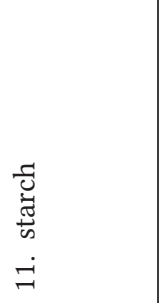 & 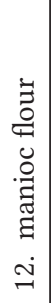 & 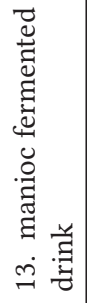 & 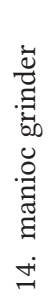 & 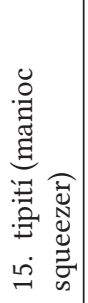 & 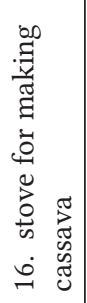 & 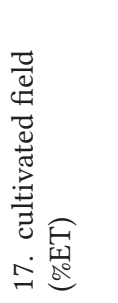 \\
\hline
\end{tabular}

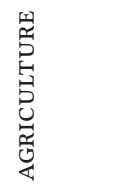




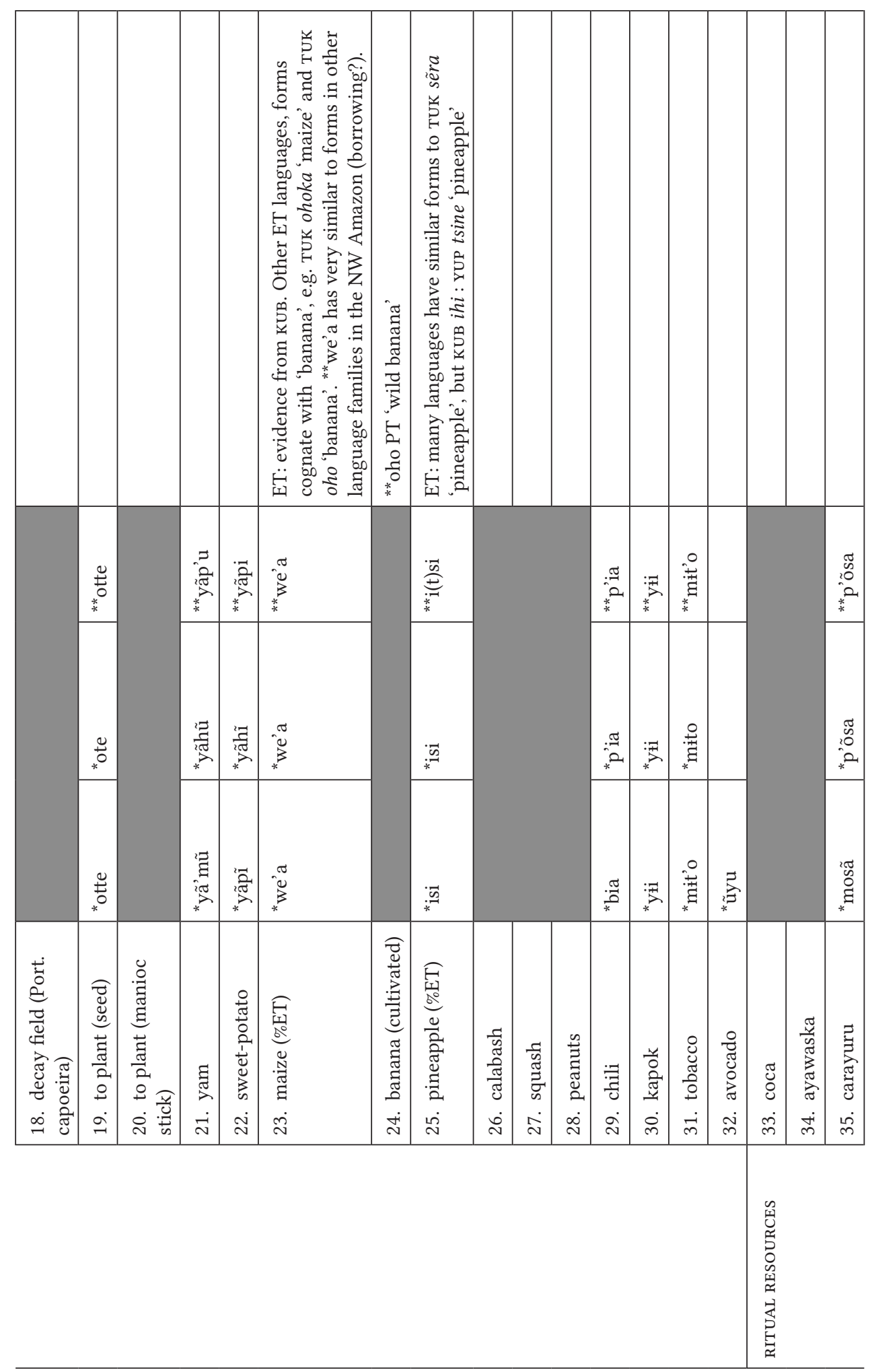




\begin{tabular}{|c|c|c|c|c|c|c|c|c|c|c|c|c|c|c|c|c|c|}
\hline & 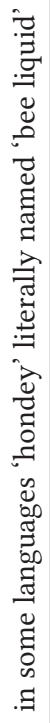 & & & & & 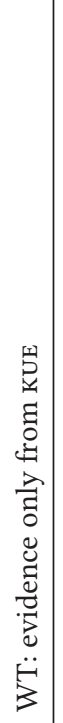 & & 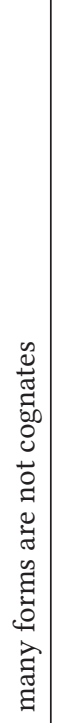 & & & & & & & & & \\
\hline & & $\begin{array}{l}\text { 早 } \\
\text { 竞 } \\
*\end{array}$ & 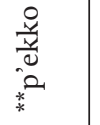 & 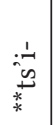 & $\mid \begin{array}{c}\mathbb{Z} \\
\underset{\mathbb{Z}}{\Xi} \\
* \\
*\end{array}$ & $\begin{array}{l}\vec{z} \\
0 \\
2 \\
* \\
*\end{array}$ & & $\begin{array}{l}\vec{y} \\
3 \\
3 \\
* \\
*\end{array}$ & 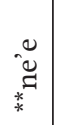 & 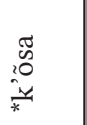 & & $\begin{array}{c}0 \\
0 \\
0.0 \\
* \\
* \\
*\end{array}$ & $\underset{*}{\frac{\pi}{\sqrt[n]{3}}}$ & 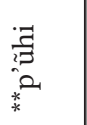 & & 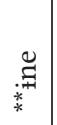 & 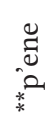 \\
\hline & $\begin{array}{l}\pi \\
*^{0} \\
*^{\circ}\end{array}$ & 总 & $\begin{array}{l}\frac{\partial}{0} \\
\text { on } \\
\text { * }\end{array}$ & $\stackrel{-1}{:-1}$ & $\mid \begin{array}{c}\mathbb{\mathscr { Z }} \\
\underset{\mathbb{\Xi}}{\Xi} \\
*\end{array}$ & $\begin{array}{l}\vec{z} \\
0 \\
0.2 \\
*\end{array}$ & & \begin{tabular}{r}
$\overrightarrow{5}$ \\
\multirow{3}{*}{} \\
$*$ \\
$*$
\end{tabular} & $\ddot{*}$ & 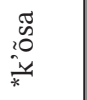 & & $\begin{array}{c}0 \\
0 \\
0 \\
* \\
*\end{array}$ & $\begin{array}{l}\text { 营 } \\
* \\
*\end{array}$ & 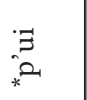 & & $\underset{*}{\stackrel{\#}{\#}}$ & $\begin{array}{l}\mathbb{E} \\
\text { E } \\
\text { * } \\
*\end{array}$ \\
\hline & $\begin{array}{l}\text { घี } \\
\text { छे } \\
*\end{array}$ & 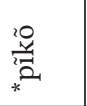 & $\begin{array}{l}\frac{8}{y} \\
\frac{y}{0} \\
\frac{\pi}{*}\end{array}$ & $: \frac{1}{\infty}$ & $\underset{*}{\stackrel{\widetilde{Z}}{\Xi}}$ & 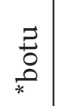 & 莺 & 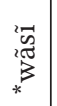 & $\ddot{\mathscr{u}}$ & $\begin{array}{l}\mathbb{W} \\
20 \\
\frac{x}{*} \\
*\end{array}$ & & \begin{tabular}{l}
0 \\
$\stackrel{\mathbb{U}}{0}$ \\
\multirow{*}{*}{}
\end{tabular} & 焉 & $\underset{*}{\stackrel{2}{\vec{\Xi}}}$ & & $\underset{i *}{\tilde{m}}$ & $\underset{*}{\mathbb{E}}$ \\
\hline 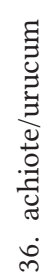 & 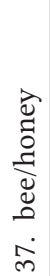 & 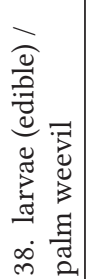 & 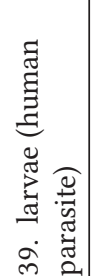 & $\begin{array}{l} \\
\infty \\
\infty \\
\dot{f} \\
\dot{q}\end{array}$ & 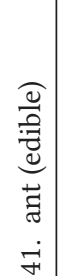 & 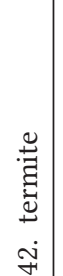 & 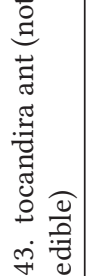 & 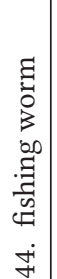 & 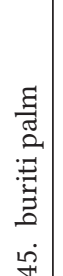 & 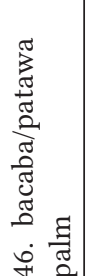 & 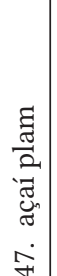 & 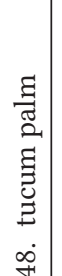 & 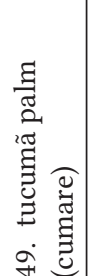 & 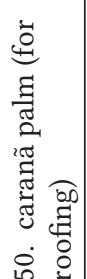 & 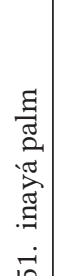 & 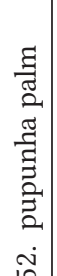 & 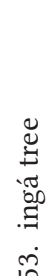 \\
\hline & 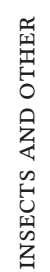 & 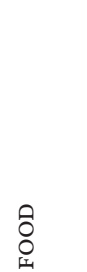 & & & & & & & 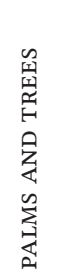 & & & & & & & & \\
\hline
\end{tabular}




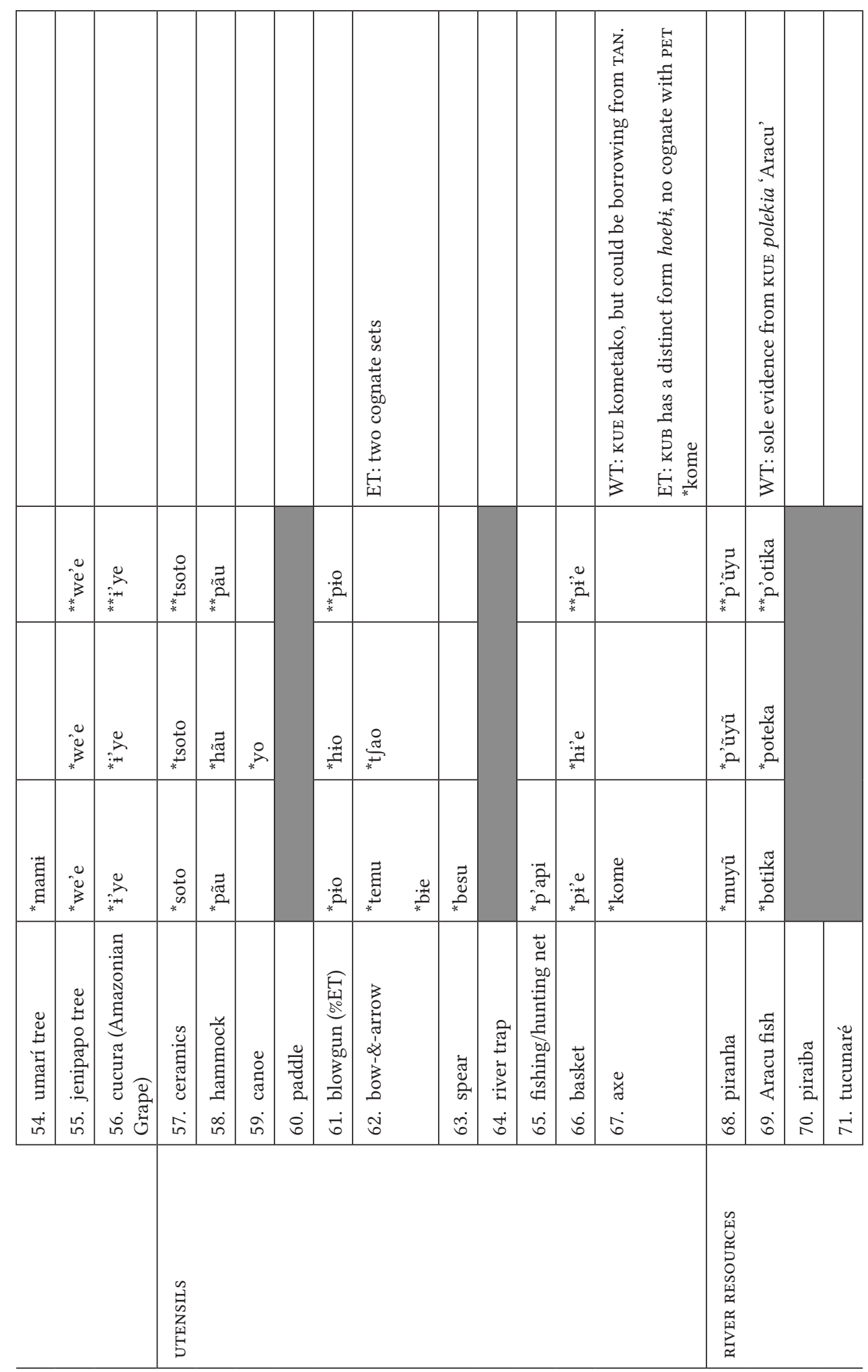




\begin{tabular}{|c|c|c|c|c|c|c|c|c|c|c|c|c|c|c|c|}
\hline & & & & & 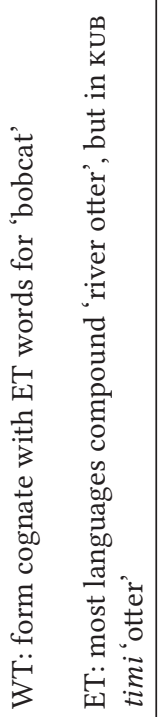 & 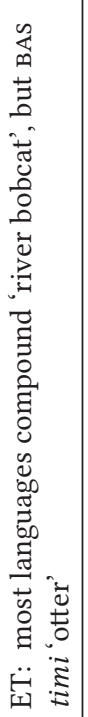 & 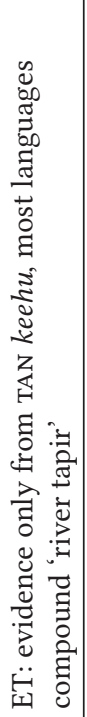 & & 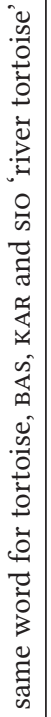 & 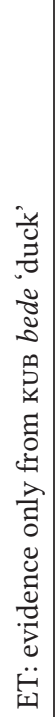 & 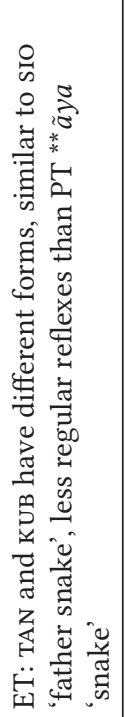 & 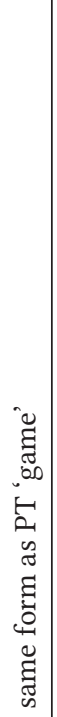 & & & \\
\hline & & 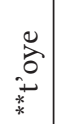 & 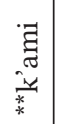 & 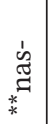 & & & 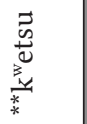 & & $\begin{array}{l}\overrightarrow{0} \\
\overrightarrow{0} \\
\vec{*} \\
* \\
*\end{array}$ & $\begin{array}{c}0 \\
0 \\
0 \\
0 \\
* \\
*\end{array}$ & & 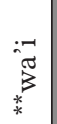 & & $\underset{*}{*}$ & 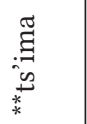 \\
\hline 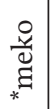 & & $\begin{array}{l}0 \\
\stackrel{0}{0} \\
\stackrel{+}{*} \\
*\end{array}$ & $\begin{array}{c}\overrightarrow{\tilde{\xi}} \\
\overrightarrow{\widetilde{\sigma}} \\
\vec{*} * \\
*\end{array}$ & $\underset{*}{\dot{q}}$ & $\underset{*}{\stackrel{0}{\pi}}$ & $\underset{*}{\stackrel{0}{\pi}}$ & 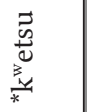 & & $\begin{array}{c}\overrightarrow{0} \\
\overrightarrow{0} \\
\vec{x} \\
\vec{k}\end{array}$ & $\begin{array}{c}0 \\
0 \\
0 \\
* \\
* \\
*\end{array}$ & & $\overline{\overrightarrow{3}}$ & & $\underset{*}{*}$ & 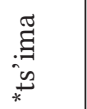 \\
\hline$\underset{\Xi}{\Xi}$ & & $\underset{*}{0}$ & 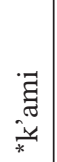 & $\underset{*}{\dot{q}}$ & 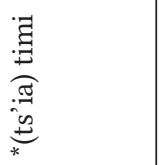 & 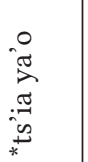 & $\begin{array}{l}\overrightarrow{\vec{n}} \\
\frac{\vec{d}}{*} \\
\frac{\vec{y}}{*}\end{array}$ & & $\begin{array}{c}\overrightarrow{0} \\
\overrightarrow{0} \\
\vec{y} \\
\vec{*}\end{array}$ & 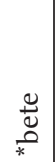 & 贯 & $\begin{array}{l}\vec{\pi} \\
\overrightarrow{3}\end{array}$ & & $\underset{*}{\otimes}$ & 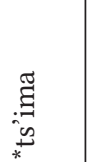 \\
\hline $\begin{array}{c}\vec{\Xi} \\
\stackrel{N}{N}\end{array}$ & 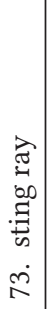 & 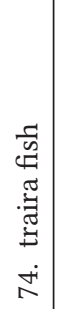 & 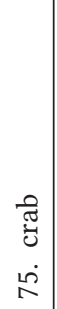 & 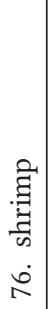 & 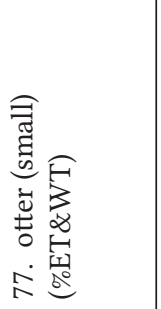 & 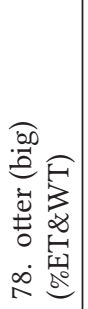 & 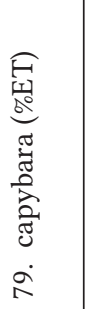 & $\begin{array}{l}\dot{0} \\
\stackrel{0}{0} \\
.00 \\
\stackrel{0}{\sigma} \\
\dot{0} \\
\infty\end{array}$ & 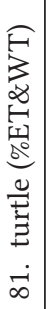 & 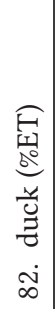 & 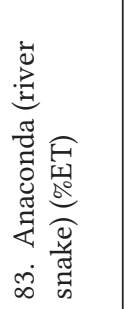 & 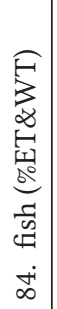 & 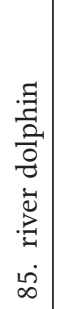 & 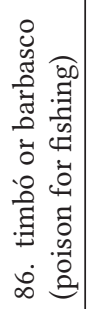 & 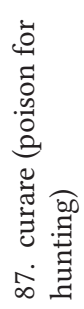 \\
\hline & & & & & & & & & & & & & & & 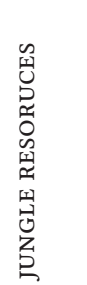 \\
\hline
\end{tabular}




\begin{tabular}{|c|c|c|c|c|c|c|c|c|c|c|c|c|c|c|c|c|c|c|c|}
\hline & & 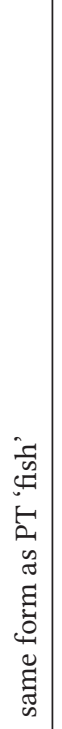 & & & & & & 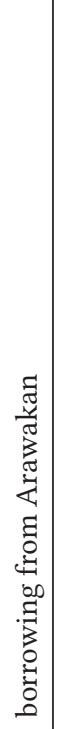 & & & 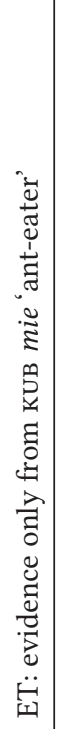 & & & 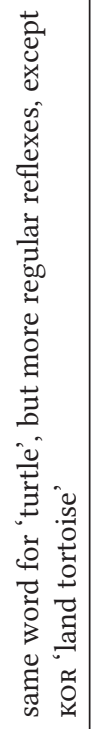 & & & & & \\
\hline 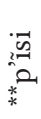 & $\begin{array}{l}\frac{\vec{y}}{\frac{y}{d}} \\
\frac{3}{3} \\
*\end{array}$ & $\begin{array}{l}-7 \\
\sqrt[\pi]{3} \\
* \\
* \\
*\end{array}$ & $\underset{*}{*}$ & \begin{tabular}{l}
$\vec{\Xi}$ \\
: \\
\multirow{2}{*}{} \\
$*$
\end{tabular} & 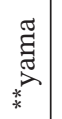 & $\underset{*}{\stackrel{0}{0}}$ & $\begin{array}{l}\underset{\mathbb{Z}}{\mathbb{Z}} \\
\underset{\mathbb{Z}}{*} \\
* \\
*\end{array}$ & & $\begin{array}{l}= \\
\overrightarrow{0} \\
3 \\
3 \\
* \\
*\end{array}$ & 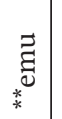 & $\underset{*}{\Xi}$ & $\begin{array}{l}= \\
\overrightarrow{7} \\
\vec{*} \\
*\end{array}$ & 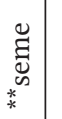 & $\begin{array}{l}\overrightarrow{0} \\
\frac{\overrightarrow{0}}{*} \\
* \\
*\end{array}$ & 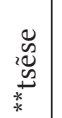 & 济 & 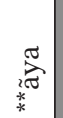 & & \\
\hline 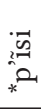 & $\begin{array}{l}\frac{i}{y} \\
\vdots \\
* \\
*\end{array}$ & 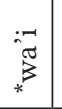 & $\stackrel{\pi}{*}$ & 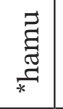 & 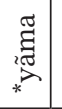 & $\underset{*}{\stackrel{0}{0}}$ & $\underset{*}{\mathbb{Z}}$ & & $\begin{array}{l}= \\
\overrightarrow{7} \\
3 \\
* \\
*\end{array}$ & $\underset{*}{\vec{\Xi}}$ & 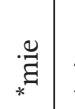 & $\begin{array}{l}\Rightarrow \\
\Rightarrow \\
\Rightarrow\end{array}$ & 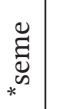 & $\begin{array}{l}\overrightarrow{0} \\
\frac{\overrightarrow{0}}{k}\end{array}$ & 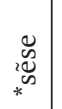 & $\begin{array}{l}i+4 \\
3 \\
*\end{array}$ & $\underset{*}{\substack{2 \pi \\
2}}$ & & I) \\
\hline 苛 & $\begin{array}{l}\frac{\pi}{3} \\
\frac{3}{3} \\
*\end{array}$ & $\begin{array}{l}-\bar{\pi} \\
n^{*} \\
*\end{array}$ & $\stackrel{\nabla}{*}$ & 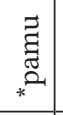 & 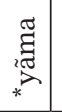 & $\underset{*}{\stackrel{0}{0}}$ & 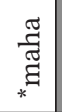 & & 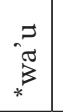 & $\underset{*}{\vec{\Xi}}$ & $\stackrel{\oiiint}{\sharp}$ & $\stackrel{\Rightarrow}{\Rightarrow}=$ & 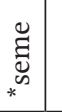 & $\begin{array}{l}\overrightarrow{0} \\
\frac{\overrightarrow{0}}{*}\end{array}$ & $\begin{array}{l}20 \\
2 \\
w \\
* \\
*\end{array}$ & $\frac{i+n}{\vec{z}}$ & 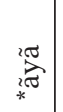 & $\stackrel{0}{20}$ & $\overbrace{*}^{\pi}$ \\
\hline 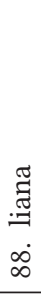 & 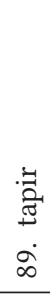 & 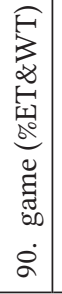 & 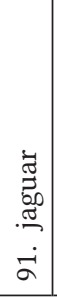 & 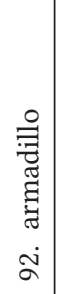 & $\begin{array}{l}\dot{\Xi} \\
\dot{v} \\
\dot{\sigma}\end{array}$ & $\begin{array}{l}\overrightarrow{\tilde{\sigma}} \\
\vec{\sigma}\end{array}$ & 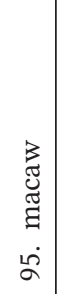 & $\begin{array}{l}\tilde{\Xi} \\
\tilde{\Xi} \\
\stackrel{0}{ } \\
\dot{\vdots}\end{array}$ & 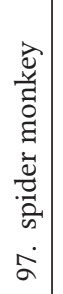 & 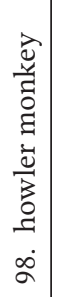 & 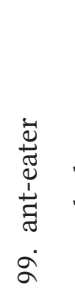 & $\begin{array}{l}\frac{5}{1} \\
\text { 음 } \\
\dot{8} \\
\dot{8}\end{array}$ & 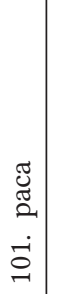 & 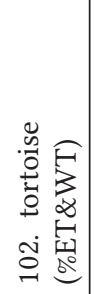 & 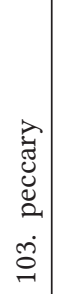 & $\begin{array}{l}: \vec{z} \\
0 \\
0 \\
\sigma \\
\dot{0} \\
\dot{0}\end{array}$ & 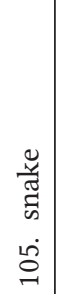 & $\begin{array}{l}0 \\
. \\
\vdots \\
\vdots \\
\dot{3} \\
\dot{0}\end{array}$ & 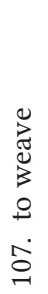 \\
\hline & & & & & & & & & & & & & & & & & & $\begin{array}{l}0 \\
z \\
2 \\
2 \\
\vdots \\
3 \\
3\end{array}$ & \\
\hline
\end{tabular}


A methodological problem, though, in interpreting pre-history from Linguistics is whether the lack of evidence for the reconstruction of a vocabulary item can be treated as absence of the trait in the society speaking the proto-language. In this study, since I am not interested exclusively in the existence of individual items in pre-historic Tukanoans, but actually in the general properties of their culture, I interpret cultural patterns, cultural changes and lack of cultural traits through a holistic structural analysis of the terminological system related to material culture. The idea of distinguishing semantic domains helps such a structural analysis, as well as paying attention to semantic relations between different items within or across semantic domains.

Another methodological problem is when two different words can be reconstructed in separate branches of family, but not up to the ProtoLanguage. Although this technically prevents us from positively saying that the proto-language had a word for such an item, it nevertheless leaves open the possibility that such items were known by the speakers of the earliest daughter languages. Another possibility, which is beyond the present state of our knowledge to test otherwise, is that the specific cultural item existed in the proto-language society, but some societies speaking daughter languages have innovated by changing the word that previously referred to the item, starting to call an older item with a new word. Again, the use of systemic structural evidence will support the postulation of the cultural item as part of the society that spoke the proto-language.

Discussion: At first, Proto-Tukanoan society can be positively characterized by having a wide knowledge of jungle resources, including a variety of edible animals, big and small game, insects, larvae, eggs, palms and trees. They definitely had some domesticated plants, including manioc, and deliberately planted crops (as indicated by the consistent cognates for the word 'to plant' **ote). They also knew ceramics, hammocks, weaved baskets and new the traditional carayuru tree, whose leafs are presently used for ceremonial painting.

In fact, this picture of Proto-Tukanoan society is very generic and is somewhat a standard, being found in other Amazonian societies, which quite directly indicates that Proto-Tukanoans shared fundamental traits with other Amazonian groups, in particular those type of groups depicted by archaeologist from the beginning of the present era (cf. section 2.1 and especially 3.1). What is more interesting, though, for studying Tukanoan pre-history are the items with more difficult reconstruction into particular branches of the family and the structural analysis of terminological systems and semantic domains.

Starting with the domain of WEAving, by observing that although a word 
for weaving must be independently reconstructed for ET and WT branches, there is strong evidence that Proto-Tukanoans knew some form of weaving, since they had hammocks, baskets and also knew the basic palms and lianas used for weaving. It is also the case that what is called 'weaving' by this paper has a variety of different words in Tukanoan languages, a few with cognates across most languages of each branch. Hence creativity in nomination and use of a variety of words to code related meanings might have helped to supplant the original PT form.

With respect to weapon terms, the blow-gun can be reconstructed for both branches of the family as * "pio-, probably derived from ** $p u$ - 'to blow' and * * $o$ 'causative. ${ }^{5}$ Reflexes of this form are found in Kubeo and WT languages, e.g. KUB pz̃oñ : sio hiowi 〈blow-gun〉 (final syllables are classifiers). In other ET languages, one finds a variant form, such as TUK bupuwi 〈blow-gun〉, where the PT root ** pu 'blow' underwent reduplication ** pu-pu> bupu. Bow and arrow can only be reconstructed independently for each branch of the family, but given that bows and arrows usually historically precede the blow-gun in different places of the world (Robert Blust p.c 2012), it is very likely that they were also known by PT society. The spear can only be reconstructed for ET, but it is a very common weapon in present WT groups (cf. Vickers 1989).

In preparation for entering into the more substantial portion of this discussion, it is important to look at a systematic pattern in Table 1. For every case a form can be reconstructed for PWT it can also be reconstructed for PET (except one case in 107 items in the word for canoe). ${ }^{6}$ This indicates that WT languages have been more conservative than ET languages regarding original terms of material culture.

Agricultural items and food processing have a complex pattern. ProtoTukanoans definitely had some knowledge of crop cultivation, as in the straightforward reconstruction of words such as 'to plant', 'yam', 'sweetpotato', 'chili', 'kapok' and 'tobacco', it seems that agriculture for them could be a less demanding and timing consuming activity than it is for present ET groups, highly specialized in manioc farming. Observing the words in

5 Synchronic changes of $\mathrm{u}>\dot{i}$ / _ o is found in Kubeo and WT languages (cf. Chacon 2012, Wheeler 1987, Johnson and Levinson 1990).

6 One might speculate why 'canoe' was reconstructed to Proto-WT, (PWT) but not to PET, especially because there is ethnographic evidence that WT groups did not used the wooden canoes until very recently (cf. Steward 1948). In the etymology of canoe terms in ET, terms for 'tree bark' are usually found, indicating a transition from a less specialized type of canoe, the one made with bark, into the current types of canoes made with logs. The word for canoe in WT languages is cognate with the classifier for long and thin objects (cf. Maihiki you 'canoe' and -yo 'classifier of long and thin objects'), so technically it is likely that in WT languages the term for the old tree bark canoes was retained and adopted to refer to the newly introduced type of wooden canoe. 
the domain of MANIOC COMPLEX, it is evident that many words found in the modern processing of manioc, such as tipiti (manioc squeezer), caxiri or chicha (manioc fermented drink) and flour cannot be reconstructed. 'Cassava' can be reconstructed, but with some inconsistent reflexes, and perhaps derived from a hypernym in PT referring to 'food', in general, which makes the reconstruction still tentative. Other utensils, such as stove and grinder, can be reconstructed for PET, but this itself is not evidence of intense manioc processing, especially because those utensils could be used for other types of food processing (DeBoer 1975, Perry 2005). The same holds for the words for starch, which can be reconstructed independently for both branches of the family, but does not exclusively code manioc processing.

The word for cultivated fields can be reconstructed to PT, but that involved a complex pattern of semantic shift in most ET languages. The form ${ }^{* *} t s$ 'io 'cultivated field' finds reflexes in all WT languages, TAN and KUB (ET): SEK s'io : KUB hio : TAN ri'o 'cultivated field'. In some ET languages the reflex appears in the name for the 'traditional hoe', an angled stick used to make holes in the ground before sticking maniocs and planting seeds, as in TUK sioga 'hoe'. In TUK, BAS, WAN and KAR, for instance, the word for 'cultivated fields' is the reflex of PT ** wese 'outdoors', which in many ET and all WT languages retained somewhat its original meaning or shifted to refer to 'sky' or 'village'. Still, in the same ET languages where ** wese shifted semantically to 'cultivated fields', the compound word for 'sky' also has a reflex of ** wese.

The analysis of the terms in the semantic fields of MANIOC COMPLEX and AGRICULTURE suggests that plants could have been cultivated at the outskirts of the house or village, being a more diversified and opportunistic activity (cf. Neves 2011), rather than the intense manioc farming, which requires the search for large fields, usually faraway from the villages, and a complex set of utensils and derived products. The absence of words for 'abandoned field' (Port. 'capoeira') in PT, 'axe' in PWT, and the complex etymology of the words referring to 'cultivated fields' may be the reflex of the development of a distinct cultivation system from the original type found in PT society.

Proto-Tukanoan's cultivation pattern must be understood in combination with the social and economic pattern inferred from the analysis of words related to JUNGLE RESOURCES, RIVER RESOURCES, UTENSILS, INSECTS AND OTHER FOODS, PALMS AND TREES. All together, the analysis of the terminology of these domains indicates that Proto-Tukanoans and perhaps the society that spoke its daughter languages until relatively recently had a more upland life orientation, rather than a riverine lifeway, as found among current ET groups, and had a more diversified economy based on a variety of jungle and small river or headwaters products.

To illustrate this analysis, first, it is striking to see that Items for JUNGLE 
RESOURCES, INSECTS AND OTHER TYPES OF FOODS, PALMS AND TREES and blowgun can be more consistently reconstructed for PT than items related to the river, agriculture and manioc complex. This simple observation may stem from a historical process where the cultural traits associated to these semantic domains have changed less so than the cultural traits associated to other semantic domains, specially agriculture and river.

Second, it is obvious that some kind of dependence on water and aquatic resources existed. A few vocabulary items common to small rivers can be straightforwardly reconstructed, such as terms for shrimp, crab, traira fish, eel, piranha and timbó or barbasco (poison for fishing). However, animals typical of large rivers, whose distribution is limited by rapids as one moves further upriver, cannot be reconstructed to any branch of the family, such as piraíba, tucunaré, sting-ray and river dolphin, reinforcing the idea of an original homeland in a headwaters environment. Fishing traps -- the most popular and elaborated fishing technique for Tukanoans - and paddle cannot be reconstructed for any branch of the family (see previous footnote for 'canoe'). Some items have a limited reconstruction, such as rapids - an important fishing site place -and fishing nets can only be reconstructed to PET, or even aracu fish, with a suspicious token in KUE, the single reflex in a WT language.

The analysis of complex etymologies also point towards the same direction. In general, the vocabulary for animals related to the river can be analyzed as marked with respect to similar animals related to the jungle. In many cases, the word for 'river' forms a compound with the noun referring otherwise to jungle animals. For instance, capybara in many ET languages is formed from the compound between 'river' and 'tapir', as in TUK dia wek $\dot{i}$ <capybara>, while the PT reconstructed form based on WT languages and TAN is ${ }^{*} k^{w}$ 'etso (the compound 'river tapir' in WT languages refer to manatee). The word for 'tortoise' can be reconstructed as ** $k$ ' $0 \dot{t}$, and in many languages the word for 〈turtle〉 is a compound translated as 〈river tortoise〉, such as in Barasano ria guu. Similar situations are found in words for big otter (Portuguese: Ariranha) with compounds such as 'river bobcat', sting ray with compounds such as 'river snake' and alligator as 'river lizard'. Anaconda has a reconstructed form for PET *pino, WT languages name it in a variety of ways, such as 'father snake' (SIO and KUE), 'river snake' (KOR) and 'big snake' (MAI). The anaconda has a very special mythological status to ET groups and not as much for WT groups.

A crucial etymology that makes this point stronger is from the analysis of words for fish, game, meat and flesh, which suggests ET groups have moved to a more riverine life-style, where hunting became less economically important than fishing (although ideologically, most ET societies still value hunting 
more than fishing). In the relation of these words, it is clear that PT word * * $w a$ ' $i$ 'game meat (or game and fish)' has changed from a hypernym in PT to mean exclusively 'fish' in ET, while it somewhat retained the original meaning in WT, such as in sio wha' $i$ : MAI bai 'meat' and 'game', Kor wa' $i$ 'meat' and 'animal (generic)'. In WT languages 'fish' is termed literally as 'small meat/ small game', as in MAI yari bai, or 'river game' as in sIo s'iaya wa' $i$ : KOR wa' $i$ yiaya. In many ET languages, 'game' is now referred to by 'great meat', as in Desano wai bigi : Barasano wai biki, while <meat has a specific term that is synonym wit <flesh〉, e.g. Tuk di'i.

While some important cultural changes can be interpreted with respect to a change from an upland, headwaters environment to a more riverine lifestyle and an economic change from a more diversified economy to a more agriculturalist economy, it is also the case that one can observe a change in the concept Tukanoans had about social spaces. While the concept of 'house' has been relatively well-preserved, notions like 'jungle', 'village', 'outdoors' and 'cultivated fields' also changed. For instance, some ET language shifted PT ** maka 'jungle' to code 'village', which is an interesting shift having in mind that reflexes of PT ** maka in WT languages code 'familiar jungle', as opposed to PWT *airo 'unfamiliar jungle'. Similarly PT ** wese 'outdoors' was shifted to mean 'cultivated field' in a few ET languages. Although I cannot offer a more intelligent observation about the terminological pattern in this domain, it is reasonable to think that changes in the social space have been part of the chain of changes documented form other domains of Tukanoan social life.

\subsection{Tukanoan Linguistic Cultural History Within the Amazonian Context}

Proto-Tukanoan Homeland: Map 1 in the introduction to this paper shows a gap between the distribution of WT and ET languages, exactly along the main stretch of Caquetá/Japurá and Putumayo rivers. In the region of the mouth of the Apaporis river into the Caquetá river, and smaller tributaries of the middle Caquetá is where we will find remnants of both ET (Tanimuka and Yupua) and WT (Kuretu) languages. On the basis of highest genetic diversity and fewer moves criterion (cf. Ehret 1976; Campbell 2004), Chacon (forthcoming) has suggested that the homeland of Proto-Tukanoan was the inter-fluvial zone between the Apaporis and Caquetá river. It is hard to be precise in what latitude that area was, but evidence suggested by the analysis of the vocabulary in this study indicates that PT speakers lived in headwaters, away from the main stream of large rivers.

In this hypothetical area, to be made more precise in future investigation, Proto-Tukanoan speakers had a cultural type that is relatively similar to the one described for the occupants of many archaeological sites in the first 
millennium AD. The types of changes noticed in the archaeological record in the beginning of the second millennium $\mathrm{AD}$, which also occurred in the Araracuara (Caquetá river) area by the same time as described by Herrera et. al (1992), might be responsible for the beginning of the cultural changes I pointed to in the analysis of Proto-Tukanoan terminological system.

Dating: While Linguistics does not provide a method for absolute dating, it is reasonable to think the diversity of the Tukanoan family as comparable to the diversity of Romance and Germanic languages, where there are languages with higher degree of mutual intelligibility (e.g. Portuguese and Spanish, Norwegian and Swedish) and languages with no mutual intelligibility (e.g. Portuguese and Sardinian, German and English). If such a parallel can be used, it is logical to think that the Tukanoan family has a time depth of about 2000-2500 years. The beginning of the diversification of the Tukanoan family roughly matches the diversification process in Amazonian societies in the beginning of the present era (Neves 2011), including the changes found in the Caquetá river area (cf. Herrera et. al. 1992). We cannot know for sure for how long Tukanoan languages have been in contact with each other after the major split between ET and WT branches, but it seems likely that the happenings in the beginning of the second millennium of the present era could have been decisive for the overall distribution of the Tukanoan languages.

Cultural Differentiation: A comparison of cultural traits between WT and ET groups, ${ }^{7}$ reveals how culturally distinct they are regarding cosmology and social and economic life. ${ }^{8}$ All ET groups have their mythological origin associated with the sub-aquatic world, as represented by the journey of an anaconda-canoe or skull of fish moving upriver, and fish-like proto-human beings that evolved into present-day humans. WT groups, on the other hand, have their mythological origin associated with the underground world, with emphasis on jungle rather than riverine traits, no reference to a mythological journey, and with long tailed proto-humans that evolved into present-day humans.

These symbolic differences bear connections with socioeconomic differences between WT and ET groups. Traditionally, ET groups have their settlements established along the rivers and fishing is the main economic activity for men. On the other hand, for WT groups hunting is the most important activity, the wooden canoe was adopted just in the beginning of

7 For all cultural information on WT groups in this section see: Steward 1948a, Beiller 1994 and Vickers 1989. For ET groups see: Goldman 1964 and 2004, Hugh-Jones 1979, Jackson 1983, Chernela 1993, Cabalzar 2008 and Cayon 2010.

8 There are certainly several common traits between both WT and ET groups, though they have not been explored systematically in the ethnological literature, nor I will attempt to do this in this paper. 
$\mathrm{XX}^{\text {th }}$ century and their houses were all distant from the larger rivers (Steward, 1948a:743). ET groups have always been characterized as intensive manioc farmers, whereas it is revealing that among the WT only the Sekoyas (Steward 1948) were said to cultivate bitter manioc in the beginning of the $X^{\text {th }}$ century. All other WT groups used to have the sweet variety of manioc. The Siona would gather the bitter variety wild and remove the poison by wrapping the pulp in bark and squeezing it (Steward 1948a:741).

As general as the characterization of these traits are, it is nevertheless significant that the cultural differences among ET and WT groups also find a systematic pattern in the reflexes of Proto-Tukanoan vocabulary, as argued in this paper. From the analysis of PT vocabulary one can also make the case that WT languages have been more conservative in the retention of words and cultural patterns that are more similar to the pattern hypothesized to PT society. Assuming that both WT and ET groups occupy present locations distinct from their original homeland in the inter-fluvial zone between the Caquetá and Apaporis river, separated by a large area nowadays occupied by a variety of other indigenous groups, it is likely that the cultural differentiation and separate geographical locations between WT and ET groups is the result of how they become distinctly integrated in different regional interethnic systems in the Northwest Amazon.

Several elements in the regional configuration of the Northwest Amazon are important to take into consideration when analyzing the pre-historic PT society and cultural differentiation process in the Tukanoan family. First, the presence of the Omagua, a Tupi-Guarani language, and their pre-Columbian ancestors, in the region occupied by WT groups. Second, the presence of Arawakan groups in the Rio Negro and surrounding areas, where ET languages are located. Third, the sorts of changes in the archaeological record observed in the Araracuara area (Caquetá river) from the beginning of the present area and its relation to the ethnographic present. Fourth, the facts related to historical happenings after the conquest, affecting population movements and socioeconomic life. A fifth element, which I cannot offer a proper treatment in this paper, is the presence of groups from smaller linguistic families or linguistic isolates in the area and their relation to Tukanoan and ProtoTukanoan peoples.

I would like to argue that the first major factor of cultural differentiation in the Tukanoan family is the result of the arrival of the archaeological culture associated with the polychrome tradition of ceramics in the Araracuara area (Caquetá river), which happened in the beginning of the second millennium of the present era, causing an intensification of agriculture and complexification of pottery decoration. By this time, WT and ET might have already differentiated in distinct branches of the family, in case our heuristic dating estimative is 
somewhat accurate. The analysis of the reconstructed PT vocabulary in this study also suggests a strong similarity between Proto-Tukanoan cultural traits and the cultural traits of the original settlers of the Araracuara area, before the appearance of the cultural traits associated with the polychrome style of ceramics. ${ }^{9}$

It is revealing that the polychrome type of pottery present in the Araracuara area has been claimed to be related to Tupian groups, which is also associated with the intensification of warfare in other areas of the Amazon (cf. Neves 2008). From map 1 above, it seems likely that WT languages were pushed up-river in the Caquetá and into the Putumayo and Napo rivers from incoming groups from the south. Spanish missionaries already found WT groups roughly in the same location where they are now (cf. Beiller 1994), so ultimately the geographic dispersal of WT languages occurred pre-historically. In addition, Goldman (1948) also describes some evidence of Tupian presence among the cultural traits of ET and neighboring Arawakan groups, and the region occupied by WT groups have been historically under influence of the Omaguan, a Tupi-Guarani language group. Hence, it is highly probable that there is a causation link between the arrival of polychrome ceramics, the complexification of material culture among Tukanoan proto-language groups and the geographical split between the two major branches of the family.

The geographical separation and subsequent happenings that affected WT and ET distinctly are the most important factors for the cultural differentiation process affecting the two branches of the Tukanoan family. WT language got in contact with the Andean foothill area as Aucas ("uncivilized" Indians for Quechua speakers), and in a relative isolation from other Amazonian groups mostly from small linguistic families or isolates - down in the Napo, Caquetá and Putumayo rivers, despite sharing similar technological resources with them. It is significant that contact between WT groups and Tupi-Guarani language groups, such as the Omaguas and Kokama, lasted until the postcolonial times, suggesting that the regional interethnic system has not changed substantially for WT language groups.

This picture contrasts with the situation of ET groups, in close contact with Arawakan language speaking groups. Quite suggestive, the cultural traits described as innovations among the ET groups bear close correspondence with neighboring Arawakan groups. Other, farther Arawakan groups also exhibit similar cultural traits, suggesting that contact with Arawakans might have caused the type of more specific cultural changes observed in the analysis of

9 This is not to suggest that Proto-Tukanoan society directly correspond to the settlers of the Araracuara area. What is suggested is that Proto-Tukanoan society shared cultural similarities with Araracuara settlers, suggesting at least a set of common cultural features shared by early inhabitants of the Northwest Amazon. 
Proto-Tukanoan vocabulary. The table below summarizes typical Arawakan cultural features (cf. Hill and Santos-Granero 2002), their resemblance to ET cultural traits and contrast with the WT pattern.

TABLE 2 - COMPARATIVE CULTURAL TRAITS AMONG ARAWAKAN,

\begin{tabular}{|l|l|l|}
\hline Arawakan & Eastern Tukanoan & Western Tukanoan \\
\hline $\begin{array}{l}\text { Continuous, flowing, } \\
\text { diasporic movements }\end{array}$ & $\begin{array}{l}\text { - Not self evident } \\
\text { - Mythology talks about } \\
\text { a journey with following } \\
\text { diasporic migrations }\end{array}$ & $\begin{array}{l}\text { No reference to } \\
\text { journey or diaspora }\end{array}$ \\
\hline $\begin{array}{l}\text { Complex system of } \\
\text { culturally and historically } \\
\text { meaningful landscapes }\end{array}$ & Similar & $\begin{array}{l}\text { No references in } \\
\text { ethnographic literature }\end{array}$ \\
\hline $\begin{array}{l}\text { Complex regional } \\
\text { interethnic networks }\end{array}$ & Similar & $\begin{array}{l}\text { Local groups and } \\
\text { settlements relatively } \\
\text { isolated }\end{array}$ \\
\hline Multilingualism & Similar & $\begin{array}{l}\text { No references in } \\
\text { ethnographic literature }\end{array}$ \\
\hline Lack of endo-warfare & $\begin{array}{l}\text { Reports of war among } \\
\text { different ET language } \\
\text { groups } \\
- \text { But not within a } \\
\text { patrilineal descent group }\end{array}$ & $\begin{array}{l}\text { Endo-warfare was } \\
\text { common even among } \\
\text { the same language } \\
\text { group }\end{array}$ \\
\hline $\begin{array}{l}\text { Romplex social hierarchy: } \\
\text { descent, ancestry, } \\
\text { consanguinity, hierarchy } \\
\text { and patrilinearity } \\
\text { sacred trumpets }\end{array}$ & Similar & $\begin{array}{l}\text { - Less complex social } \\
\text { stratification and } \\
\text { kinship system } \\
- \text { More lax patrilineal } \\
\text { ideology } \\
\boldsymbol{-} \text { Reports of } \\
\text { uxorilocality (Maihuna) }\end{array}$ \\
\hline Riverine lifeway & Similar & $\begin{array}{l}\text { Emphasis on land } \\
\text { resources }\end{array}$ \\
\hline Similar & $\begin{array}{l}\text { No references in } \\
\text { ethnographic literature }\end{array}$ \\
\hline
\end{tabular}




\section{ET AND WT LANGUAGES}

Intensification of the contact between ET and Arawakan groups, and the formative period of the Upper Rio Negro regional subsystem, leading to the cultural convergence of ET and Arawakan groups, can be relatively dated after the major geographical split of WT and ET, suggesting that ET languages moved into the Pira-Parana and Vaupés river in a later time, perhaps pushed by the same reason that WT groups were pushed to the west, having Arawakan and other local nomadic groups (Nadahup - formerly Makú - plus Kakua and Nukak) as the original inhabitants of the area (cf. Koch-Grünberg 2005[1909], Nimuendajú 1950, Wright 1992).

Adding to table 2 above, one could also argue that ET groups became more specialized in manioc farming as the result of contact with Arawakan groups. Another possibility is the suggestion that the pattern of intense manioc cultivation throughout the Amazon might have been caused by social-cultural changes brought by European colonization (cf. Denevan 2001). If this is right, ET people might have first shifted to a more riverine lifestyle and then, in a later time during the colonial period, shifted to a more intense cultivation of bitter manioc, although manioc was probably cultivated or known by ProtoTukanoans since a long time ago.

\section{Conclusion}

This paper has proposed a reconstruction of 107 items from a sample of basic material culture of indigenous groups in the Northwestern Amazon. By the pattern of reconstructions, etymological analysis and attention to systematic patterns in the terminological systems, we were able to assess some cultural traits that speakers of Proto-Tukanoan had and to demonstrate an important cultural differentiation process that occurred between the ET and WT branches of the family. This type of change, as I argued, might be related to the distinct ways WT and ET language groups were integrated in the distinct regional subsystem in Northwestern Amazon. There is likely a correlation between the cultural differentiation among ET and WT languages, the emergence of the regional system in the Vaupes area, the happenings around the beginning of the second millennia of the present era as documented in the archaeological record, and the consequences brought by the colonial period. We are just starting to make sense of such complex events that shaped the life of Tukanoans and the Northwestern Amazon in the last millennia.

\section{References}

Barasana Literacy Committee, Paula S. Jones and Wendell H. Jones (compilers). 2009. Diccionario bilingüe: Eduria and Barasana-Español, Español-Eduria and Barasana. 
Bogotá: Editorial Fundación para el Desarrollo de los Pueblos Marginados.

Barnes, Janet. 1999. Tucano. In: R.M.W. Dixon and Alexandra Y. Aikhenvald, The Amazonian Languages (eds.), pp: 207-226. Cambridge: Cambridge University Press.

Bellier, Iréne. 1994. Los Maihuna: Tucano Occidentales. In: , Santos, Fernando and Frederica Barclay (eds.) Guia Etnográfico del Alto-Amazonas. Volume 1. Ulpiano Páez: Quito.

Bellwood, Peter and Colin Renfrew (eds.). 2003. Examining the Farming/Language Dispersal Hypothesis. Cambridge: McDonald Institute for Archaeological Research.

Blomster, Jeffrey P., Hector Neff, and Michael D. Glascock. 2005. Olmec Pottery Production and Export in Ancient Mexico Determined Through Elemental Analysis. Science 307:1078-1072.

Blust, Robert. 1976. Austronesian Culture History: Some Linguistic Inferences and Their Relations to the Archaeological Record. World Archaeology, Vol. 8, No. 1, Archaeology and Linguistics, pp. 19-43.

Campbell, Lyle. 2004. Historical Linguistics: an introduction. 2nd edition. The MIT Press: Cambridge Massachussets.

Campbell, Lyle, Terrence Kaufman and Thomas C. Smith-Stark. 1986. Meso-America as a Linguistic Area. Language 62(3):530-571.

Campbell, Lyle and Terry Kaufman. 1976. A Linguistic Look at the Olmecs. American Antiquity, v. 41, n. 1. 80-89.

Chacon, Thiago. forthcoming. A revision of Proto-Tukano Consonant Reconstruction and Tukanoan Family Classification. Accepted for publicaton with minor reviews at the International fournal of American Linguists. University of Chicago.

Chernela, Janet. 1993. The Wanano Indians of the Brazilian Amazon: A Sense of Space. Austin: University of Texas Press.

Cook, Dorothy M. and Frances L. Gralow. 2001. Diccionario bilingüe KoreguajeEspañol, Español-Koreguaje. Bogotá: Editorial Alberto Lleras Camargo. 203p.

DeBoer, Warren. 1975. The Archaeological Evidence for Manioc Cultivation: A Cautionary Note. American Antiquity 40(4):419-433.

Denevan, William. 2001. Cultivate Landscapes of Native Amazonia and the Andes. Oxford University Press.

Ehret, Christopher. 1976. Linguistic Evidence and its correlation with Archaelogy. World Archaelogy 8(1):5-18

Goldman, Irving. 1948. Tribes of the Vaupés-Caquetá region. In: Julian H. Steward (ed.), Handbook of South American Indians, vol. 3: The tropical tribes, , 763-98. Washington: Bureau of American Ethnology. 1964. The Cubeo: Indians of the Northwest Amazon. University of Illinois Press. 2004. Cubeo Hehenawa: Methaphysics of a Northwestern Amazonian People. New York: Columbian University Press.

González de Pérez, Maria Stella, and Rodríguez de Montes, Maria Luisa eds. 2000. 
Lenguas Indígenas de Colombia, una visión descriptiva. Santafé de Bogotá: Instituto Caro y Cuervo.

Hackenberg, Michal, James B. Petersen and Eduardo Goes Neves. 1999. Village Size and Permanence in Amazonia: Two Archaeological Examples from Brazil. Latin American Antiquity 10(4):353-276.

Herrera, Luisa Fernanda, Ines Cavelier, Camilo Rodriguez, Santiago Mora. 1992. The technical Transformation of an Agricultural System in the Colombian Amazon. World Archaeology, 24:1 The Humid Tropics, pp. 98-113.

Hill, Jonathan D. and Fernando Santos-Granero. 2002. Introduction. In: Jonathan Hill and Fernando Santos-Granero (eds.), Comparative Arawakan Histories: Rethinking Language Family and Culture Area in Amazonia, Urbana: The University of Illinois.

Hornborg, Alf. 2005 Ethnogenesis, Regional Integration, and Ecology in Prehistoric Amazonia: Towards a Systemic Perspective, Current Anthropology 46(4):589-620. Chicago.

Hornborg, Alf and Jonathan D. Hill. 2011. Introduction: Ethnicity in Ancient Amazonia. In: Alf Hornborg and Jonathan D. Hill (eds.), Ethnicity in Ancient Amazonia: Reconstructing Past Identities from Archaeology, Linguistics and Ethnohistory. Boulder: The University of Colorado Press.

Huber, Randal Q. and Robert B. Reed (compilers). 1992. Vocabulario comparativo: Palabras selectas de lenguas indígenas de Colombia. Bogotá: Instituto Lingüístico de Verano.

Hugh-Jones, Christine. 1979. From the milk river: spatial and temporal processes in the Northwest Amazon. Cambridge: Cambridge University Press.

Jackson, Jean.1983. The Fish People. Linguistic exogamy and Tukanoann identity in Northwest Amazonia. Cambridge: Cambridge University Press.

Johnson, Orville and Stephen Levinson 1990. Gramática secoya. Cuadernos Etnolinguísticos 11. Quito: Instituto Linguístico de Verano.

Justeson, John S. 1986. The origin of Writing Systems: Preclassic Mesoamerica. World Archaelogy, v.17, n.3, Early Writing Systems, pp. 437-458.

Kaufman, Terry and John S Justeson. 1993. A Decipherment of Epi-Olmec Hieroglyphic Writing. Science v. 259:1703-1711.

Kirch, P. 2000. On the Road of the Winds: An Archaeological History of the Pacific Islands before European Contact. University of California Press, Berkeley.

Koch-Grünberg, Theodor. 2005 [1909]. Dois Anos entre os Indígenas: Viagens no noroeste do Brasil [Two years among the Indians: Travels in the Brazilian Northwest] (1903/1905). EDUA and FSDB: Manaus.

1913. Betoya-Sprachen Nordwestbrasiliens und der angrenzenden Gebiete. Anthropos, VIII.

1914. Betoya-Sprachen Nordwestbrasiliens und der angrenzenden Gebiete. Anthropos, IX. 
Lathrap, Donald 1970 The Upper Amazon, Ancient Peoples and Places. London: Thames and Hudson. .

Mallroy, J. P. 1991. In search of the Indo-Europeans: Language, Archaeology and Myth. Londo: Thames and Hudson.

Malone, T. 1987. Proto-Tucanoan and Tucanoan genetic relationship. Colombia: Instituto Linguistico de Verano. [Ms.]

Metzger, Ronald G. (compiler). 2000. Marĩ yaye mena Carapana, yaia yaye mena Español macãrĩcã tuti = Carapana-Español, diccionario de 1000 palabras. Bogotá: Editorial Alberta Lleras Camargo.

Michael, Lev, Christine Beier, Stephanie Farmer, Gregory Finley, and John Sylak. 2012. Diccionario Bilingüe Máíjíki-Castellano y Castellano-Máíjíki (versión agosto 2012). Máíhîki Project internal document. [Ms.]

Morse, Nancy L. Jay K. Salser and Neva Salser (compilers). 1999. Diccionario ilustrado bilingüe: cubeo-español español-cubeo. Bogotá: Editorial Alberto Lleras Camargo.

Neves EG. 2008. Ecology, ceramic chronology and distributions, long-term history, and political change in the Amazonian floodplain. In: Silverman $\mathrm{H}$ and Isbell $\mathrm{W}$ (eds.), Handbook of South American Archaeology. New York: Springer.

. 2011. Archaeological Cultures and Past Identities in the Pre-Colonial Central Amazon. In: Alf Hornborg and Jonathan D. Hill (eds.) Ethnicity in Ancient Amazonia: Reconstructing Past Identities from Archaeology, Linguistics and Ethnohistory. Boulder: The University of Colorado Press.

Nimuendajú, Curt. 1950 [1927]. Reconhcimento dos rios Içana, Aiary e Uaupés. Journal de la Société des Américanistes. Tome 39:125-182.

Oliver J. 2008. The archaeology of agriculture in ancient Amazonia. In: Silverman H and Isbell W, Handbook of South American Archaeology. New York: Springer.

Perry, Linda. 2005. Reassessing the Traditional Interpretation of "Manioc" Artifacts in the Orinoco Valley of Venezuela. Latin American Antiquity 16(4):209-426.

Piaguaje, Ramón, Elías Piaguaje, Orville E. Johnson, and Mary Johnson, compilers. 1992. Vocabulario Secoya. Quito: Instituto Lingüístico de Verano.

Pye, Mary E. y John E. Clark. 2006 Los Olmecas son Mixe-Zoques: Contribuciones de Gareth W. Lowe a la arqueología del Formativo. In: J.P. Laporte, B. Arroyo y H. Mejía (eds.), XIX Simposio de Investigaciones Arqueológicas en Guatemala, 2005 ), pp.70-82. Museo Nacional de Arqueología y Etnología, Guatemala (versión digital).

Ramirez, Henri. 1997a. A fala Tukano dos Ye’pâ-Masa: Tomo II: dicionário. Manaus: Inspetoria Salesiana Missionaria da Amazônia: CEDEM.

Rodrigues, Aryon. 1964. A classificação do tronco linguistico Tupi. Revista Anthropologica 12: 99-107. São Paulo.

Ross, Malcom, Andrew Pawley and Meredith Osmond. 2007. The Lexicon of ProtoOceanic: The culture and environment of Proto-Oceanic Society. v. 2 Physical Environment. 
Sharer, Robert J., Robert J. Sharer, Andrew K., Balkansky, James H. Burton, Gary M. Feinman, Kent V. Flannery, David C. Grove, Joyce Marcus, Robert G. Moyle, T. Douglas Price, Eisa M. Redmond, Robert G. Reynolds, Prudence M. Rice, Charles S. Spencer, James B. Stoltman, and Jason Yaeger . 2006 On The Logic Of Archaeological Inference: Early Formative Pottery And The Evolution Of Mesoamerican Societies. Latin American Antiquity 17(1):90-103.

Schurr, Theodore. 2004. The peopling of the New World. Prespectives from Molecular Anthropology. Annual Review of Anthropology 33, pp. 551-583.

Smothermon, Jeffrey R. and Josephine H. Smothermon (compilers). 1993. Masa ye, gawa ye rãca ãmara tuti (Macuna - español diccionario de 850 palabras). Bogotá: Editorial Alberto Lleras Camargo. 206 p.

Steward, Julian H. 1948a. Western Tucanoan Tribes. In: Julian Steward (ed.), Handbook of South American Indians, vol. 3, pp. 737-749.Washington: Smithsonian Institution. . 1948b. Culture Areas of the Tropical Forests. In: Julian Steward (ed.), Handbook of South American Indians, vol. 3, pp. 737-748. Washington: Smithsonian Institution.

Velie, Daniel and Virginia Velie. 1981. Vocabulario orejón. Lima: Ministerio de Educación and Instituto Lingüístico de Verano.

Vickers, William T. 1989. Los Sionas y Sekoyas: su adaptación al ambiente amazônico. Abya Yala: Quito.

Waltz, Nathan and Alva Wheeler. 1972. Proto-Tucanoan. In: Esther Matteson (ed.), Comparative Studies in Amerindian Languages, pp. 19-49. The Hague: Mouton.

Waltz, Nathan E., (compiler), Paula Simmons de Jones and Carolyn de Waltz (editors). 2007. Diccionario bilingüe: Wanano o Guanano-Español, Español-Wanano o Guanano. Bogotá, D.C.: Editorial Fundación para el Desarrollo de los Pueblos Marginados.

Wheeler, Alva. 1987b. El pueblo siona del río Putumayo, Colombia. vol. II: Diccionario. Bogotá: Instituto Linguístico de Verano.

Wilson, Warren and D. L. Dufour. 2002. Why "Bitter" Cassava? Productivity of "Bitter" and "Sweet" Cassava in a Tukanoan Indian Settlement in the Northwest Amazon. Economic Botany 56:1: 49-57.

Wright, Robin. 1992. História Indígena do Noroeste Amazônico: Hipóteses, Questões e Perspectivas. In: Manuela Carneiro da Cunha (ed.), História dos Índios no Brasil, São Paulo: Fundação Amparao à Pesquisa do Estado de São Paulo/Companhia das Letras/Secretaria Municipal de Cultura.

Data recebimento: 01/12/2012

Data aceite: 27/05/2013 